\title{
Review Article \\ The Roles of Traditional Chinese Medicine: Shen-Fu Injection on the Postresuscitation Care Bundle
}

\author{
Qian Zhang and Chunsheng Li \\ Department of Emergency Medicine, Beijing Chaoyang Hospital, Capital Medical University, \\ 8 Workers' Stadium South Road, Chaoyang District, Beijing 100020, China \\ Correspondence should be addressed to Chunsheng Li; lcscyyy@163.com
}

Received 3 December 2012; Revised 20 May 2013; Accepted 31 July 2013

Academic Editor: Ka Kit Hui

Copyright (C) 2013 Q. Zhang and C. Li. This is an open access article distributed under the Creative Commons Attribution License, which permits unrestricted use, distribution, and reproduction in any medium, provided the original work is properly cited.

\begin{abstract}
Survival rates following in-hospital and out-of-hospital cardiac arrests remain disappointingly low. Organ injury caused by ischemia and hypoxia during prolonged cardiac arrest is compounded by reperfusion injury that occurs when a spontaneous circulation is restored. A bundle of procedures, which may need to be administered simultaneously, is required. The procedures include prompt identification and treatment of the cause of cardiac arrest, as well as a definitive airway and ventilation together. Additional benefit is possible with appropriate forms of early goal-directed therapy and achieving therapeutic hypothermia within the first few hours, followed by gradual rewarming and ensuring glycaemic control to be within a range of 6 to $10 \mathrm{mmol} / \mathrm{L}$. All these would be important and need to be continued for at least 24 hours. Previous studies have showed that the effects of Shen-Fu injection (SFI) are based on aconitine properties, supplemented by ginsenoside, which can scavenge free radicals, improve energy metabolism, inhibit inflammatory mediators, suppress cell apoptosis, and alleviate mitochondrial damage. SFI, like many other complex prescriptions of traditional Chinese medicine, was also found to be more effective than any of its ingredient used separately in vivo. As the postresuscitation care bundle is known to be, the present paper focuses on the role of SFI played on the postresuscitation care bundle.
\end{abstract}

\section{Introduction}

Cardiac arrest (CA) results in whole-body ischemia/reperfusion and represents the most severe shock state, during which the delivery of oxygen and metabolic substrates is abruptly halted, and metabolites are no longer removed. Cardiopulmonary resuscitation (CPR) only partially reverses this process [1]. Furthermore, the myocardium is a main target tissue during this form of ischemia/reperfusion, and the destruction of tissue progresses even after circulation has been successfully restored.

In patients who initially achieve return of spontaneous circulation (ROSC) after CA, the significant subsequent morbidity and mortality are largely due to the cerebral and cardiac dysfunction that accompanies prolonged whole-body ischemia. CA contributes to hemodynamic disorders that cause the systemic release of massive oxygen free radical, lactic acid, and metabolites of arachidonic acid, which could reach the different tissues by blood circulation and cause ischemia/reperfusion injury $[2,3]$. Inadequate tissue oxygen delivery can persist even after ROSC; accumulated oxygen debt leads to activation of immunologic pathways and systemic inflammation, which increases the risk of multiple organ failure. This condition had many features in common with sepsis [4]. The pathophysiological changes cause multiple organ dysfunctions and have recently been termed the "postcardiac arrest syndrome," which comprises anoxic brain injury, postcardiac arrest myocardial dysfunction, systemic ischemia/reperfusion response, and persistent precipitating pathology [5].

Shen-Fu injection (SFI), which has been commonly used in China for nearly 800 years, is a well-known traditional Chinese medical formulation containing ginseng (Panax; family: Araliaceae) and fuzi (Radix aconiti lateralis preparata, Aconitum carmichaeli Debx.; family: Ranunculaceae), which is produced using multistage countercurrent extraction and macroporous resin adsorption technology (Yảan Sanjiu Pharmaceutical Co., Ltd., China). Its quality is controlled strictly 
according to the standard of China Ministry of Public Health and fingerprint technology was adopted in the process of production to ensure its quality [6]. Their chemical structures are as shown in Figure 1.

Lots of clinical and epidemiological studies have demonstrated that SFI has significant protective effects on ischemia/reperfusion injury of the brain, spinal cord, kidney, intestine, liver, and especially the heart [7-11]. However, relatively little is known about the effects of SFI on postresuscitation care bundle. The present paper focuses on the association between postresuscitation care bundle and SFI and explains the roles of SFI on the postresuscitation care bundle treatment.

\section{Postresuscitation Care Bundles}

Bundles are a group of "therapies" built around the best evidence-based guidelines, which, when implemented together, produce greater benefit in terms of outcome than the individual therapeutic interventions [12]. The conception of care bundles has been proposed based on the holistic principle that the whole is greater than the sum of its parts. Bundles play a useful role to help remove the constraints imposed by these deviations and variations by means of constructing the elements into packages that must be implemented in strict compliance for every patient, at each and every single time to ensure uniformity as well as universality [13].

In the year of 2005, the final ring of the chain of survival was updated to reflect the importance of postresuscitation care in determining the ultimate outcome following CA [14]. Organ injury caused by ischemia and hypoxia during prolonged $\mathrm{CA}$ is compounded by reperfusion injury that occurs when a spontaneous circulation is restored. These insults trigger a systemic inflammatory response, similar to that associated with sepsis [15]. Here, we propose a "postresuscitation care bundle" which comprises (1) early coronary reperfusion and hemodynamic optimization, (2) airway and ventilation management, (3) therapeutic hypothermia, (4) neurological enhancement measures and monitoring, and (5) glycaemic control [16].

\subsection{Early Coronary Reperfusion and Hemodynamic Optimiza-} tion. Patients resuscitated from CA who have electrocardiographic criteria for ST-elevation myocardial infarction should undergo immediate coronary angiography, with subsequent percutaneous coronary intervention (PCI) if indicated. If PCI is not available, thrombolytic therapy is an appropriate alternative for postcardiac arrest management of ST-elevation myocardial infarction [17]. This may also be helpful for monitoring blood pressures during therapeutic hypothermia and for accurate measurement of hemodynamic parameters so as to determine the most appropriate combination of medications for maintenance of perfusion. Central venous pressure monitoring would also be useful.

2.2. Airway and Ventilation Management. Airway control is crucial in the initial stages of postresuscitation management.
Insertion of a definitive airway (if not done yet) guided by capnography is followed by a chest radiograph for confirmation of tube position. While mechanical ventilation would be required so as to reduce the work of breathing, the rate of ventilation and tidal volume would need to be adjusted in order to maintain arterial oxyhemoglobin saturation at $\geq 94 \%[18,19]$. The concerns that hyperoxaemia during the reperfusion phase after ROSC with $100 \%$ oxygen may lead to increased brain lipidperoxidation, increased metabolic dysfunction, and neurological degeneration, as well as concerns about its impact on short-term functional outcome, have resulted in calls of ventilation with room air or an inspired oxygen fraction titrated to maintain a pulse oximetry reading of $94 \%$ to $98 \%$ [20]. Weaning the patients from $100 \%$ oxygen to the $\mathrm{FiO}_{2}$ required to maintain $\mathrm{SpO}_{2}$ at the above stated levels should begin once ROSC is achieved [21].

2.3. Therapeutic Hypothermia. It has been well demonstrated that brain temperatures during the first 24 hours after ROSC have a significant effect on survival and neurological recovery in patients who remain comatose soon after ROSC [22]. Preclinical and clinical evidence strongly supports mild therapeutic hypothermia as an effective therapy for the postcardiac arrest syndrome [23]. Unconscious adult patients with spontaneous circulation after out-of-hospital VF cardiac arrest should be cooled to $32-34^{\circ} \mathrm{C}$ for at least 12 to 24 hours. Induced hypothermia might also benefit unconscious adult patients with spontaneous circulation after out-of-hospital cardiac arrest from a nonshockable rhythm or in-hospital cardiac arrest [24].

\subsection{Neurological Enhancement Measures and Monitoring.} Postcardiac arrest brain injury is a result of initial ischemic injury followed by reperfusion injury occurring within the hours and days after ROSC [25]. Features indicating occurrence of brain injury in post-ROSC patients include coma, seizures, myoclonus, and various degrees of neurocognitive dysfunction, ranging from memory deficits to a persistent vegetative state and finally brain death. The neurological prognosis in the majority of comatose CA survivors cannot be reliably predicted until at least 72 hours after CPR. Currently, the principal neuroprotective measures recommended include normoventilation with controlled oxygenation to avoid hyperoxaemia and minimize the likelihood of lowering cerebral perfusion or aggravating cerebral ischemia, achieving normoglycaemia to optimize neuronal recovery and therapeutic hypothermia to minimize the cerebral and multisystem metabolic functions until biochemical and cellular parameters are better optimized [26].

2.5. Glycaemic Control. Hyperglycaemia occurring postROSC has been associated with increased mortality and worse neurological outcomes [27]. Similarly, hypoglycaemia is also associated with poor outcomes in critically ill patients [28]. The strategy is to maintain blood sugar levels at 6$10 \mathrm{mmol} / \mathrm{L}$. Blood glucose concentrations must be monitored frequently in these patients, and hyperglycemia should be treated with an insulin infusion. A target glucose range with 


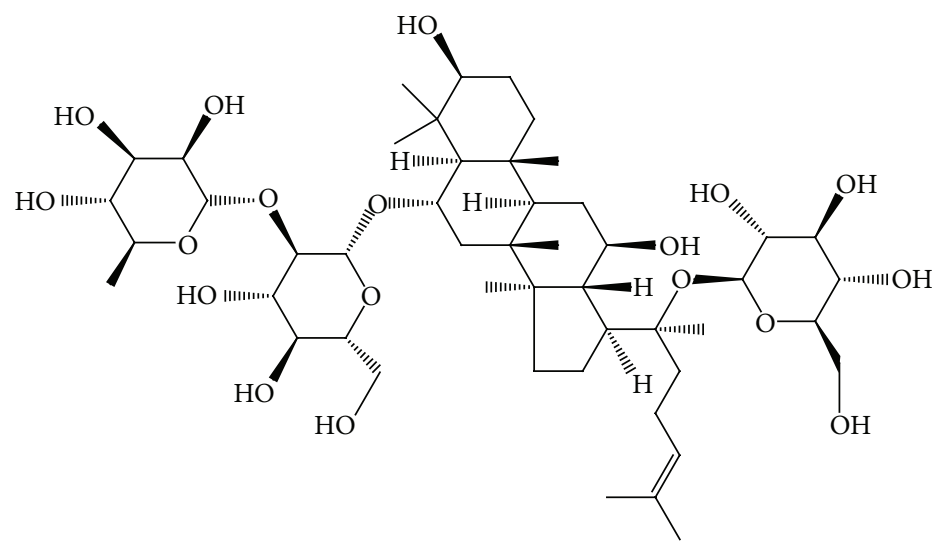

Ginsenoside-Re

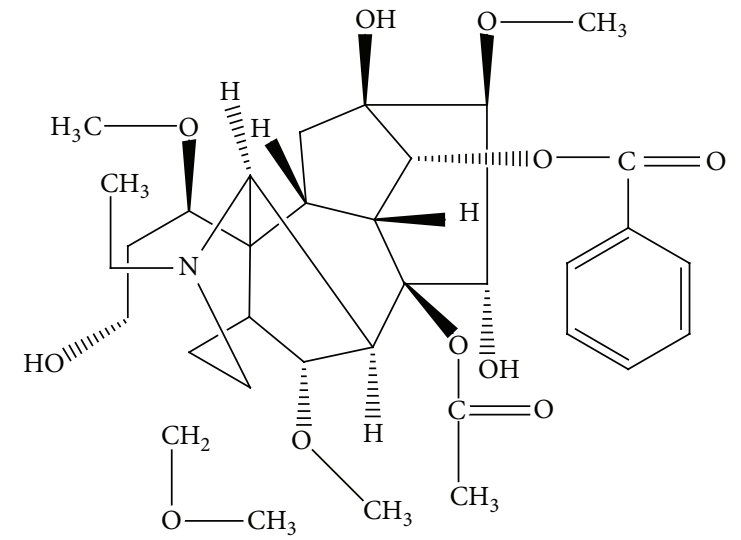

Aconitine

FIgURE 1: The chemical structures of ginsenoside-Re and Aconitine.

an upper value of $8.0 \mathrm{mmol} / \mathrm{L}(144 \mathrm{mg} / \mathrm{dL})$ has been suggested by others [29-31]. The lower value of $6.1 \mathrm{mmol} / \mathrm{L}(110 \mathrm{mg} / \mathrm{dL})$ may not reduce mortality any further but instead may expose patients to the potentially harmful effects of hypoglycemia [29]. The incidence of hypoglycemia in another recent study of intensive insulin therapy exceeded $18 \%$, and some have cautioned against its routine use in the critically ill patients [32-34]. Regardless of the chosen glucose target range, blood glucose must be measured frequently, especially when insulin is started and during cooling and rewarming periods.

\section{The Roles of SFI on the Postresuscitation Care Bundle Treatment}

3.1. SFI Mitigates the Postresuscitation Myocardial Dysfunction. Postresuscitation myocardial dysfunction has been implicated as one of the major causes of fatal outcomes in patients who fail to survive hospitalization after CPR [35]. Results from Ji et al. showed that SFI significantly increased mean arterial pressure and improved the cardiac output and ejection fraction after ROSC. SFI also can attenuate postresuscitation myocardial dysfunction through beneficial effects on energy metabolism and remarkable antioxidant capacity. Meanwhile, it is also demonstrated that SFI can prevent and cure different kinds of arrhythmia (tachycardia and bradycardia), while such two-way regulating effect has rarely been found in western medicine [36]. Furthermore, in an in vitro study, $\mathrm{Gu}$ et al. reported the cardioprotective effects of SFI by decreasing myocardial injury, improving myocardial ultrastructure, inhibiting Bcl-2, Bax, and caspase 3 expressions, and reducing myocardial apoptosis [37].

3.2. SFI Extenuates Postresuscitation Lung Injury. Since postROSC patients are at risk of acute lung injury or acute respiratory distress syndrome, the standard recommendation for ventilation would be normocapnia. Excessive tidal volumes would not also be recommended owing to the risks of increased intrathoracic pressures with attendant reduced venous return and cardiac output. Zhang et al. have reported that SFI resulted in improving oxygenation index, respiratory index, oxygen extraction ratio, dynamic lung compliance, airway resistance, external vascular lung water index, and pulmonary vascular permeability index, which indicates that SFI can effectively protect pulmonary gas exchange function, increase oxygen consumption and extraction, decrease structural lung damage, and alleviate pulmonary edema after ROSC [38]. A recent study has also demonstrated that SFI could reduce postresuscitation lung injury and improve lung immune function by regulating lung imbalance of Th1/Th2 [39].

3.3. SFI Alleviates Postresuscitation Cerebral Injury. In an in vitro study, Yang et al. showed that cerebral injury is aggravated progressively at the early phase following CPR. SFI could alleviate cerebral injury after $\mathrm{CPR}$, and this protective effect might be of dosage effect relationship. Large dosage of SFI could promote the expression of neuron-specific enolase in neurons, which might alleviate the cerebral injury after CPR [40].

3.4. SFI and Glycaemic Control. Hyperglycemia is common after CA due to the upregulated stress response. Tight blood glucose control remains controversial in the critical care setting. It has been shown to improve survival in a surgical intensive care setting, but this outcome did not convey to the medical intensive care setting [41]. The blood glucose concentration should be monitored frequently in the postcardiac arrest syndrome patient, especially when instituting the therapeutics hypothermia. In an in vitro study, Shan-shan et al. reported that SFI could increase insulin secretion by increasing PI3K expression, which can improve hyperglycemia during CPR. The effects were likely caused by increased PI3K contents in islets. The mechanism may be partly related to SFI reversing the disequilibrium of oxidization and antioxidation [42]. 


\section{The Holistic Approach with SFI}

4.1. The Effect of SFI on $\mathrm{Na}^{+}-\mathrm{K}^{+}$-ATPase/Ca ${ }^{2+}$-ATPase Activity. $\mathrm{Na}^{+}-\mathrm{K}^{+}$-ATPase activity is an electrogenic process in which two $\mathrm{Na}^{+}$ions extrude out of the cell, whereas one $\mathrm{K}^{+}$ion enters the cell, thereby maintaining an appropriate transmembrane $\mathrm{Na}^{+}$gradient. $\mathrm{Ca}^{2+}$-ATPase enzyme is another sarcolemmal enzyme. Intracellular calcium loading is considered to represent the common denominator of ischemia/reperfusion-induced cell dysfunction and death. Various studies showed that $\mathrm{Na}^{+}-\mathrm{K}^{+}$-ATPase and $\mathrm{Ca}^{2+}$ ATPase enzymes may play a key role in the prevention of ischemia/reperfusion [43-45]. Luo et al. have found that SFI could restore the ability of $\mathrm{Na}^{+}-\mathrm{K}^{+}$-ATPase and $\mathrm{Ca}^{2+}$-ATPase enzyme activities, and it may be one of the mechanisms by which SFI could attenuate the postresuscitation myocardial dysfunction [46]. Additionally, the effect of SFI's blockage on the sodium channels in cardiac myocytes may be one of the important molecular mechanisms for its cardiac active effectiveness.

4.2. Effects of SFI on Oxygen Free Radicals. CPR can be viewed as a process of whole-body ischemia/reperfusion [47]. A large amount of oxygen free radicals (OFRs) produced during the ischemia/reperfusion play a major role in myocardial damage. Malondialdehyde (MDA) is an end product of lipid peroxidation that causes cellular damage and disruption of cell membranes when tissue antioxidants are exhausted. MDA has been found to increase in myocardial tissue after myocardial ischemia/reperfusion [48]. Superoxide dismutase (SOD) is a metalloenzyme that catalyzes the dismutation of $\mathrm{O}_{2}{ }^{-}$into $\mathrm{O}_{2}$ and $\mathrm{H}_{2} \mathrm{O}_{2}$ and affords protection against OFRs damage [49]. The activity of SOD could reflect the in vivo scavenging capability of OFRs. Reducing of MDA content and attenuating the decrease of SOD activities in myocardial tissue have cardioprotective effects [50]. A recent study has shown that, comparing with saline group, A recent study has shown that the MDA content was significantly decreased in SFI-treated myocardium subjected to ischemia/reperfusion injury. At the same time, the activities of serum SOD in the SFI group were significantly higher than in the saline group [51]. These results indicate that SFI can reduce MDA content, attenuate the decrease of SOD activities in myocardial tissue, and thus alleviate the degree of myocardial injury.

4.3. SFI Mitigates the Impact of Calcium Overload. $\mathrm{Ca}^{2+}$ is a ubiquitous signal for regulating cellular function, including survival and death [52]. While a small amount of $\mathrm{Ca}^{2+}$ is necessary for the optimal physiological function of the heart, growing evidence suggests that an increased cytosolic free $\mathrm{Ca}^{2+}$ overload is one of the major contributors of myocardial injury induced by ischemia/reperfusion [53-55]. Therefore, $\mathrm{Ca}^{2+}$ handling in the postischemic myocardium has become a prime target. A recent study showed that the protective effects of SFI on myocardial ischemia/reperfusion injury were realized by repressing the opening of $\mathrm{Ca}^{2+}$ channel myocardial cell membrane, reducing inflow of $\mathrm{Ca}^{2+}$, inhibiting calcium overload, decreasing OFRs, and suppressing inflammation [56]. These results indicate that SFI can mitigate the impact of calcium overload.

4.4. The Relationship between SFI and PI3K/Akt Signaling Pathway. Some researches have showed that PI3K/Akt signaling pathway plays a crucial role in protecting the myocardium from ischemia/reperfusion injury [57, 58]. Strong evidence shows that endothelial nitric oxide synthase (eNOS) is an important target of Akt and eNOS plays the role of an important cardiovascular protective molecule [59]. Recent studies show that increased expression of Akt and eNOS alleviates the ischemia/reperfusion injury [60, 61]. Wu et al. showed that SFI treatment resulted in an enhanced level eNOS which was significantly higher than that of saline-treated ischemia/reperfusion group [62]. These results suggested that SFI-induced cardioprotective effects are mediated through Akt-induced eNOS phosphorylation.

4.5. SFI Prevents Cardiomyocytes Apoptosis. In a present study, after 24 hours post-ROSC, significant myocardial damage and apoptosis emerged, accompanied by increased protein expression of $\mathrm{Bcl}-2, \mathrm{Bax}$, and active caspase- 3 [63]. The most principal pathways for apoptosis initiation are termed the Bcl-2/Bax-controlled pathway. Furthermore, downregulation of $\mathrm{Bcl}-2 / \mathrm{Bax}$ expression after postresuscitation might result in the activation of the caspase family of proteases, such as caspase 3 , which is responsible for the induction of apoptotic cell death, leading to internucleosomal DNA fragmentation [64]. In an in vitro study, Wang et al. reported that treatment with different doses of SFI protected cardiac myocyte cultures from hypoxia/reoxygenation-induced apoptosis. Caspase-3 activation was decreased in hypoxic/reoxygenated cardiomyocytes cotreated with SFI when compared to hypoxia/reoxygenation alone treated cultures. Expression of the $\mathrm{Bcl}-2$ proteins was increased in SFI-treated cardiomyocytes subjected to hypoxia/reoxygenation [65]. These results showed that SFI could significantly attenuate postresuscitation myocardial dysfunction by modulating apoptosis.

\section{Conclusion}

In summary, postcardiac arrest syndrome is associated with complex pathophysiological changes and a high mortality rate, and a multidisciplinary approach to treatment is optimal. SFI might be useful in the treatment of postcardiac arrest syndrome, incorporating the multilayer and multitarget advantages of TCM. Above all, SFI plays a key role during postresuscitation care bundle. Furthermore, with the initiation of large-scale, multicenter studies and further research into the pharmacological actions of SFI, we believe that SFI will eventually be widely used for postresuscitation care bundle treatment. 


\section{Conflict of Interests}

The authors declare that they have no commercial associations or sources of support that might pose a conflict of interests.

\section{Acknowledgment}

The Shen-Fu injection was supplied by the Yaan Sanjiu Pharmaceutical Co., Ltd., China.

\section{References}

[1] J. Herlitz, J. Engdahl, L. Svensson, K.-A. Ängquist, J. Silfverstolpe, and S. Holmberg, "Major differences in 1-month survival between hospitals in Sweden among initial survivors of out-ofhospital cardiac arrest," Resuscitation, vol. 70, no. 3, pp. 404409, 2006.

[2] I. Jacobs, V. Nadkarni, J. Bahr et al., "Cardiac arrest and cardiopulmonary resuscitation outcome reports: update and simplification of the Utstein templates for resuscitation registries. A statement for healthcare professionals from a task force of the International Liaison Committee on Resuscitation (American Heart Association, European Resuscitation Council, Australian Resuscitation Council, New Zealand Resuscitation Council," Circulation, vol. 110, no. 21, pp. 3385-3397, 2004.

[3] I. G. Stiell, G. A. Wells, B. Field et al., "Advanced cardiac life support in out-of-hospital cardiac arrest," The New England Journal of Medicine, vol. 351, no. 7, pp. 647-656, 2004.

[4] C. Adrie, M. Adib-Conquy, I. Laurent et al., "Successful cardiopulmonary resuscitation after cardiac arrest as a "sepsis-like" syndrome," Circulation, vol. 106, no. 5, pp. 562-568, 2002.

[5] D. F. Gaieski, B. S. Abella, and M. Goyal, "CPR and postarrest care: overview, documentation, and databases," Chest, vol. 141, no. 4, pp. 1082-1089, 2012.

[6] Z. H. Zhu, L. Z. Xiong, H. L. Dong, W. N. Hu, X. L. Zeng, and L. C. Hou, "Dose response effects of Shenfu injection on ischemic reperfusion injury of spinal cord in rabbit," Chinese Journal of Anesthesiology, vol. 20, pp. 664-668, 2000.

[7] Z.-Y. Xia, L.-Y. Zhan, Y.-H. He, and X.-Y. Liu, "The effect of Shen-Fu on gastrointestinal tract injury and its potential mechanism during cardio-pulmonary bypass in patients undergoing cardiac surgery," Chinese Journal of Traumatology, vol. 6, no. 4, pp. 245-248, 2003.

[8] X. Y. Liu, H. D. Zou, J. F. Yu, H. B. Huang, and G. X. Xiong, "Protective effect of Shen-fu injection on multiple organ damage in rabbit during ischemia reperfusion," Chinese Journal of Anesthesiology, vol. 17, pp. 430-432, 1997.

[9] B.-J. Zhang, Y.-L. Wang, C.-Y. Wang, and J.-J. Ke, “Effect of Shenfu injection on nuclear factor- $\kappa \mathrm{B}$ during myocardial ischemia/reperfusion injury in rats," Chinese Journal of Traumatology, vol. 8, no. 4, pp. 200-204, 2005.

[10] T. P. Zhang and M. Zhao, "Protective effect of Shen-Fu injection on heart injury induced by ischemia/reperfusion," Journal of Molecular and Cellular Cardiology, vol. 42, pp. S209-S210, 2007.

[11] Z. R. Tang, "Effect of Shen-fu on hemodynamics of hypovolemic shock andoxygen delivery," Chinese Journal of Pathophysiology, vol. 21, no. 10, pp. 1954-1957, 2005.

[12] R. G. Masterton, "Sepsis care bundles and clinicians," Intensive Care Medicine, vol. 35, no. 7, pp. 1149-1151, 2009.
[13] M. M. Levy, P. J. Pronovost, R. P. Dellinger et al., "Sepsis change bundles: converting guidelines into meaningful change in behavior and clinical outcome," Critical Care Medicine, vol. 32, no. 11, pp. S595-S597, 2004.

[14] S. J. Fletcher and A. C. Quinn, “The surviving sepsis campaign and sepsis care bundles: substance or sophistry?" Anaesthesia, vol. 61, no. 4, pp. 313-315, 2006.

[15] C. Adrie, I. Laurent, M. Monchi, A. Cariou, J. F. Dhainaou, and C. Spaulding, "Postresuscitation disease after cardiac arrest: a sepsis-like syndrome?” Current Opinion in Critical Care, vol. 10, no. 3, pp. 208-212, 2004.

[16] J. P. Nolan and J. Soar, "Post resuscitation care-time for a care bundle?” Resuscitation, vol. 76, no. 2, pp. 161-162, 2008.

[17] I. Laurent, M. Monchi, J.-D. Chiche et al., "Reversible myocardial dysfunction in survivors of out-of-hospital cardiac arrest," Journal of the American College of Cardiology, vol. 40, no. 12, pp. 2110-2116, 2002.

[18] L. N. Tremblay and A. S. Slutsky, "Ventilator-induced lung injury: from the bench to the bedside," Intensive Care Medicine, vol. 32, no. 1, pp. 24-33, 2006.

[19] L. B. Ware and M. A. Matthay, "The acute respiratory distress syndrome," The New England Journal of Medicine, vol. 342, no. 18, pp. 1334-1349, 2000.

[20] J. P. Muizelaar, A. Marmarou, J. D. Ward et al., "Adverse effects of prolonged hyperventilation in patients with severe head injury: a randomized clinical trial," Journal of Neurosurgery, vol. 75, no. 5, pp. 731-739, 1991.

[21] R. Knafelj, P. Radsel, T. Ploj, and M. Noc, "Primary percutaneous coronary intervention and mild induced hypothermia in comatose survivors of ventricular fibrillation with ST-elevation acute myocardial infarction," Resuscitation, vol. 74, no. 2, pp. 227-234, 2007.

[22] A. Zeiner, M. Holzer, F. Sterz et al., "Hyperthermia after cardiac arrest is associated with an unfavorable neurologic outcome," Archives of Internal Medicine, vol. 161, no. 21, pp. 2007-2012, 2001.

[23] D. F. Gaieski, R. A. Band, B. S. Abella et al., "Early goaldirected hemodynamic optimization combined with therapeutic hypothermia in comatose survivors of out-of-hospital cardiac arrest," Resuscitation, vol. 80, no. 4, pp. 418-424, 2009.

[24] J. P. Nolan, P. T. Morley, T. L. Vanden Hoek et al., "Therapeutic hypothermia after cardiac arrest. An advisory statement by the Advanced Life Support Task Force of the International Liaison Committee on Resuscitation," Resuscitation, vol. 57, no. 3, pp. 231-235, 2003.

[25] R. R. Noppens, R. F. Kelm, R. Lindemann, K. Engelhard, C. Werner, and O. Kempski, "Effects of a single-dose hypertonic saline hydroxyethyl starch on cerebral blood flow, long-term outcome, neurogenesis, and neuronal survival after cardiac arrest and cardiopulmonary resuscitation in rats," Critical Care Medicine, vol. 40, no. 7, pp. 2149-2156, 2012.

[26] J. Hovdenes, J. H. Laake, L. Aaberge, H. Haugaa, and J. F. Bugge, "Therapeutic hypothermia after out-of-hospital cardiac arrest: experiences with patients treated with percutaneous coronary intervention and cardiogenic shock," Acta Anaesthesiologica Scandinavica, vol. 51, no. 2, pp. 137-142, 2007.

[27] A. Kennedy and J. Soar, "Management of Glucose post cardiac arrest," http://www.bestbets.org/bets/bet.php?id=1043.

[28] Y. M. Arabi, H. M. Tamim, and A. H. Rishu, "Hypoglycemia with intensive insulin therapy in critically ill patients: predisposing factors and association with mortality," Critical Care Medicine, vol. 37, no. 9, pp. 2536-2544, 2009. 
[29] G. van den Berghe, A. Wilmer, G. Hermans et al., "Intensive insulin therapy in the medical ICU," The New England Journal of Medicine, vol. 354, no. 5, pp. 449-461, 2006.

[30] T. Oksanen, M. B. Skrifvars, T. Varpula et al., "Strict versus moderate glucose control after resuscitation from ventricular fibrillation," Intensive Care Medicine, vol. 33, no. 12, pp. 20932100, 2007.

[31] H. Losert, F. Sterz, R. O. Roine et al., "Strict normoglycaemic blood glucose levels in the therapeutic management of patients within $12 \mathrm{~h}$ after cardiac arrest might not be necessary," Resuscitation, vol. 76, no. 2, pp. 214-220, 2008.

[32] S. J. Finney, C. Zekveld, A. Elia, and T. W. Evans, "Glucose control and mortality in critically Ill patients," Journal of the American Medical Association, vol. 290, no. 15, pp. 2041-2047, 2003.

[33] F. M. Brunkhorst, C. Engel, F. Bloos et al., "Intensive insulin therapy and pentastarch resuscitation in severe sepsis," The New England Journal of Medicine, vol. 358, no. 2, pp. 125-139, 2008.

[34] P. E. Marik and J. Varon, "Intensive insulin therapy in the ICU: is it now time to jump off the bandwagon?" Resuscitation, vol. 74, no. 1, pp. 191-193, 2007.

[35] C.-Y. Hsu, C.-H. Huang, W.-T. Chang et al., "Cardioprotective effect of therapeutic hypothermia for postresuscitation myocardial dysfunction," Shock, vol. 32, no. 2, pp. 210-216, 2009.

[36] X.-F. Ji, L. Yang, M.-Y. Zhang, C.-S. Li, S. Wang, and L.-H. Cong, "Shen-Fu injection attenuates postresuscitation myocardial dysfunction in a porcine model of cardiac arrest," Shock, vol. 35, no. 5, pp. 530-536, 2011.

[37] W. Gu, C. Li, W. Yin, Z. Guo, X. Hou, and D. Zhang, "Shenfu injection reduces postresuscitation myocardial dysfunction in a porcine model of cardiac arrest by modulating apoptosis," Shock, vol. 38, no. 3, pp. 301-306, 2012.

[38] M.-Y. Zhang, X.-F. Ji, S. Wang, and C.-S. Li, "Shen-Fu injection attenuates postresuscitation lung injury in a porcine model of cardiac arrest," Resuscitation, vol. 83, no. 9, pp. 1152-1158, 2012.

[39] W. Gu, C. Li, W. Yin, X. Hou, and D. Zhang, "Effects of shenfu injection on the expression of $t$-cell-specific transcription factors t-bet/gata-3 in porcine postresuscitation lung injury," Evidence-Based Complementary and Alternative Medicine, vol. 2013, Article ID 464650, 8 pages, 2013.

[40] F. J. Yang, Y. S. Zheng, D. X. Li, and W. L. Deng, "Effect of Shenfu injection on micro-circulation," Journal of Biomedical Engineering, vol. 20, pp. 91-94, 2003 (Chinese).

[41] G. van den Berghe, P. Wouters, F. Weekers et al., "Intensive insulin therapy in critically ill patients," The New England Journal of Medicine, vol. 345, no. 19, pp. 1359-1367, 2001.

[42] T. Shan-shan, S. Min, and K. Wei, "Effect of insular phosphatidy linositol 3kinase on shenfu injection improving hyperglycemia in rabbits undergoing cardiopulmonary bypass," Journal of Clinical Anaesthesiology, vol. 26, no. 4, 2010.

[43] S.-Y. Zheng, J. Sun, X. Zhao, and J.-G. Xu, "Protective effect of Shen-Fu on myocardial ischemia-reperfusion injury in rats," American Journal of Chinese Medicine, vol. 32, no. 2, pp. 209220, 2004.

[44] S. M. Krause, W. E. Jacobus, and L. C. Becker, "Alterations in cardiac sarcoplasmic reticulum calcium transport in the postischemic 'stunned' myocardium," Circulation Research, vol. 65, no. 2, pp. 526-530, 1989.

[45] M. S. Kim and T. Akera, "O2 free radicals: cause of ischemiareperfusion injury to cardiac $\mathrm{Na}^{+}-\mathrm{K}^{+}$-ATPase," The American journal of physiology, vol. 252, no. 2, part 2, pp. H252-H257, 1987.
[46] J. Luo, S. Min, K. Wei, and J. Cao, "Ion channel mechanism and ingredient bases of Shenfu Decoction's cardiac electrophysiological effects," Journal of Ethnopharmacology, vol. 117, no. 3, pp. 439-445, 2008.

[47] L. Wiklund, H. S. Sharma, and S. Basu, "Circulatory arrest as a model for studies of global ischemic injury and neuroprotection," Annals of the New York Academy of Sciences, vol. 1053, pp. 205-219, 2005.

[48] M. V. Cohen, "Free radicals in ischemic and reperfusion myocardial injury: is this the time for clinical trials?" Annals of Internal Medicine, vol. 111, no. 11, pp. 918-931, 1989.

[49] K. H. Haider and W. H. Stimson, "Cardiac myofibrillar proteins: biochemical markers to estimate myocardial injury," Molecular and Cellular Biochemistry, vol. 194, no. 1-2, pp. 31-39, 1999.

[50] H. Esterbauer, R. J. Schaur, and H. Zollner, "Chemistry and Biochemistry of 4-hydroxynonenal, malonaldehyde and related aldehydes," Free Radical Biology and Medicine, vol. 11, no. 1, pp. 81-128, 1991.

[51] S.-Y. Zhen, J.-G. Xu, and Z.-Z. Zhao, "The protective effect of shenfu injection on myocardium against ischemia reperfusion injury in rats," Chinese Journal of Integrated Traditional and Western Medicine, vol. 24, no. 6, pp. 541-544, 2004.

[52] L. Chen, X.-Y. Lu, J. Li, J.-D. Fu, Z.-N. Zhou, and H.-T. Yang, "Intermittent hypoxia protects cardiomyocytes against ischemia-reperfusion injury-induced alterations in $\mathrm{Ca}^{2+}$ homeostasis and contraction via the sarcoplasmic reticulum and $\mathrm{Na}^{+} / \mathrm{Ca}^{2+}$ exchange mechanisms," American Journal of Physiology. Cell Physiology, vol. 291, no. 5, p. C1099, 2006.

[53] R. B. Singh and N. S. Dhalla, "Ischemia-reperfusion-induced changes in sarcolemmal $\mathrm{Na}^{+} / \mathrm{K}^{+}$-ATPase are due to the activation of calpain in the heart," Canadian Journal of Physiology and Pharmacology, vol. 88, no. 3, pp. 388-397, 2010.

[54] H. K. Saini-Chohan and N. S. Dhalla, "Attenuation of ischemiareperfusion-induced alterations in intracellular $\mathrm{Ca}^{2+}$ in cardiomyocytes from hearts treated with $\mathrm{N}$-acetylcysteine and N-mercaptopropionylglycinel," Canadian Journal of Physiology and Pharmacology, vol. 87, no. 12, pp. 1110-1119, 2009.

[55] H. Gao, L. Chen, and H.-T. Yang, "Activation of $\alpha 1 \mathrm{~B}-$ adrenoceptors alleviates ischemia/reperfusion injury by limitation of mitochondrial $\mathrm{Ca}^{2+}$ overload in cardiomyocytes," Cardiovascular Research, vol. 75, no. 3, pp. 584-595, 2007.

[56] W.-H. Zhang, S.-B. Fu, F.-H. Lu et al., "Involvement of calciumsensing receptor in ischemia/reperfusion-induced apoptosis in rat cardiomyocytes," Biochemical and Biophysical Research Communications, vol. 347, no. 4, pp. 872-881, 2006.

[57] P. H. Sugden, "Ras, akt, and mechanotransduction in the cardiac myocyte," Circulation Research, vol. 93, no. 12, pp. 1179-1192, 2003.

[58] B. D. Manning and L. C. Cantley, "AKT/PKB signaling: navigating downstream," Cell, vol. 129, no. 7, pp. 1261-1274, 2007.

[59] S. V. Penumathsa, M. Thirunavukkarasu, S. M. Samuel et al., "Niacin bound chromium treatment induces myocardial Glut4 translocation and caveolar interaction via Akt, AMPK and eNOS phosphorylation in streptozotocin induced diabetic rats after ischemia-reperfusion injury," Biochimica et Biophysica Acta, vol. 1792, no. 1, pp. 39-48, 2009.

[60] F. Gao, E. Gao, T.-L. Yue et al., "Nitric oxide mediates the antiapoptotic effect of insulin in myocardial ischemia-reperfusion: the roles of PI3-kinase, Akt, and endothelial nitric oxide synthase phosphorylation," Circulation, vol. 105, no. 12, pp. 1497-1502, 2002. 
[61] R. Schulz, M. Kelm, and G. Heusch, "Nitric oxide in myocardial ischemia/reperfusion injury," Cardiovascular Research, vol. 61, no. 3, pp. 402-413, 2004.

[62] Y. Wu, Z.-Y. Xia, Q.-T. Meng et al., "Shen-fu injection preconditioning inhibits myocardial ischemia-reperfusion injury in diabetic rats: activation of eNOS via the PI3K/Akt pathway," Journal of Biomedicine and Biotechnology, vol. 2011, Article ID 384627, 9 pages, 2011.

[63] E. Palojoki, A. Saraste, A. Eriksson et al., "Cardiomyocyte apoptosis and ventricular remodeling after myocardial infarction in rats," American Journal of Physiology. Heart and Circulatory Physiology, vol. 280, no. 6, pp. H2726-H2731, 2001.

[64] L. Scorrano and S. J. Korsmeyer, "Mechanisms of cytochrome c release by proapoptotic BCL-2 family members," Biochemical and Biophysical Research Communications, vol. 304, no. 3, pp. 437-444, 2003.

[65] Y.-L. Wang, C.-Y. Wang, B.-J. Zhang, and Z.-Z. Zhang, "Shenfu injection suppresses apoptosis by regulation of $\mathrm{Bcl}-2$ and caspase-3 during hypoxia/reoxygenation in neonatal rat cardiomyocytes in vitro," Molecular Biology Reports, vol. 36, no. 2, pp. 365-370, 2009. 


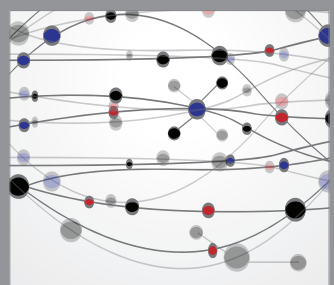

The Scientific World Journal
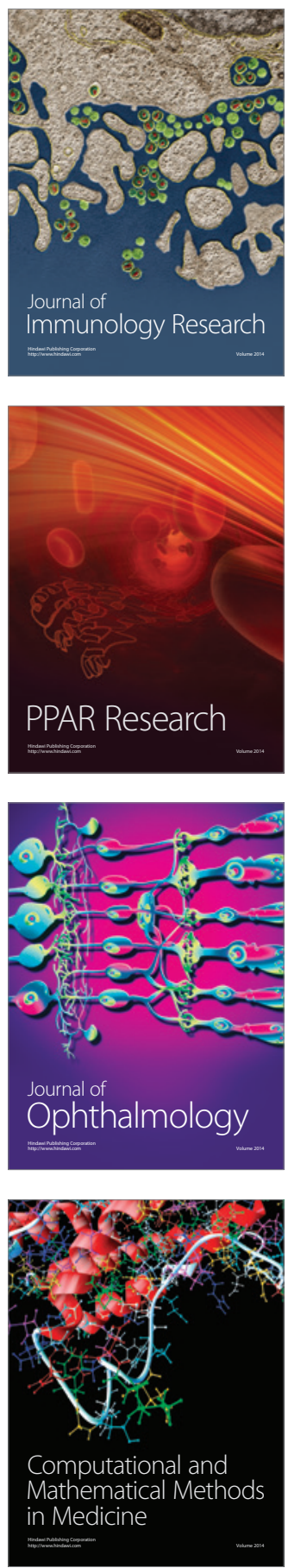

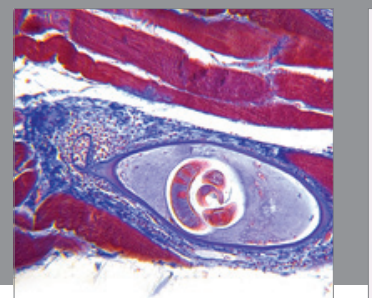

Gastroenterology

Research and Practice
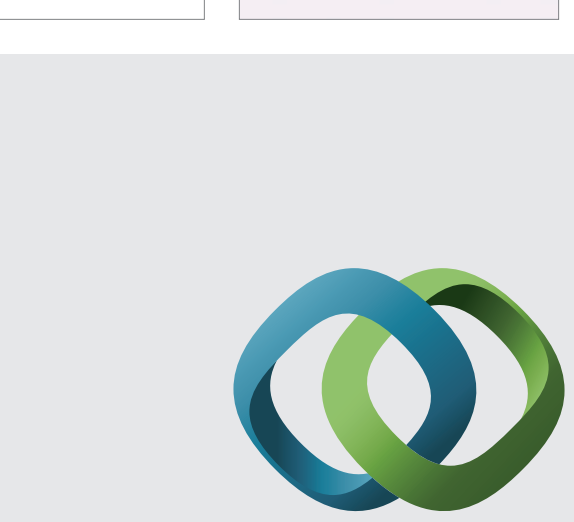

\section{Hindawi}

Submit your manuscripts at

http://www.hindawi.com
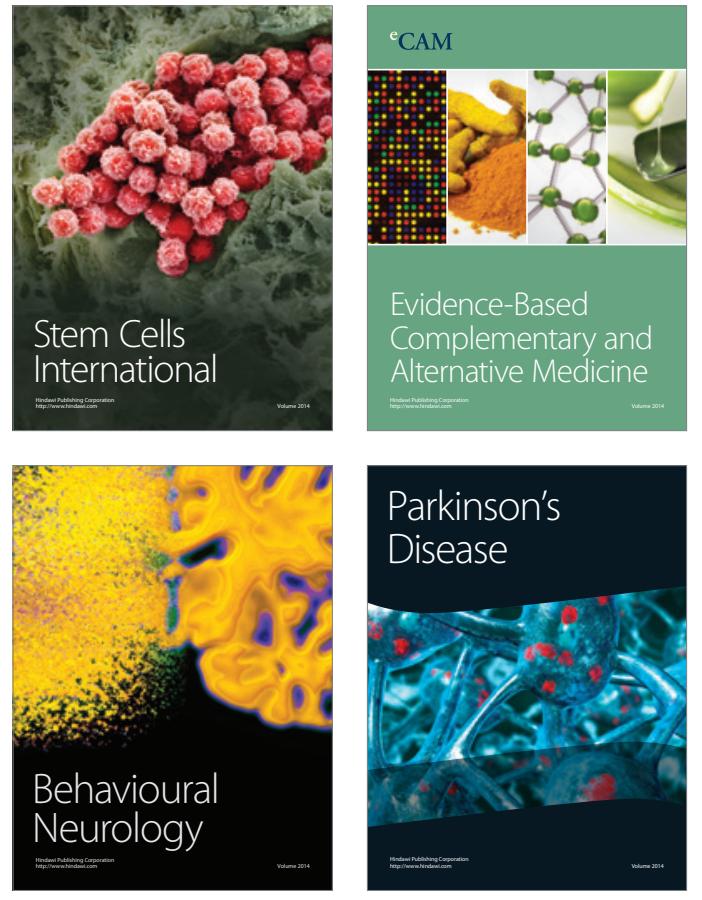
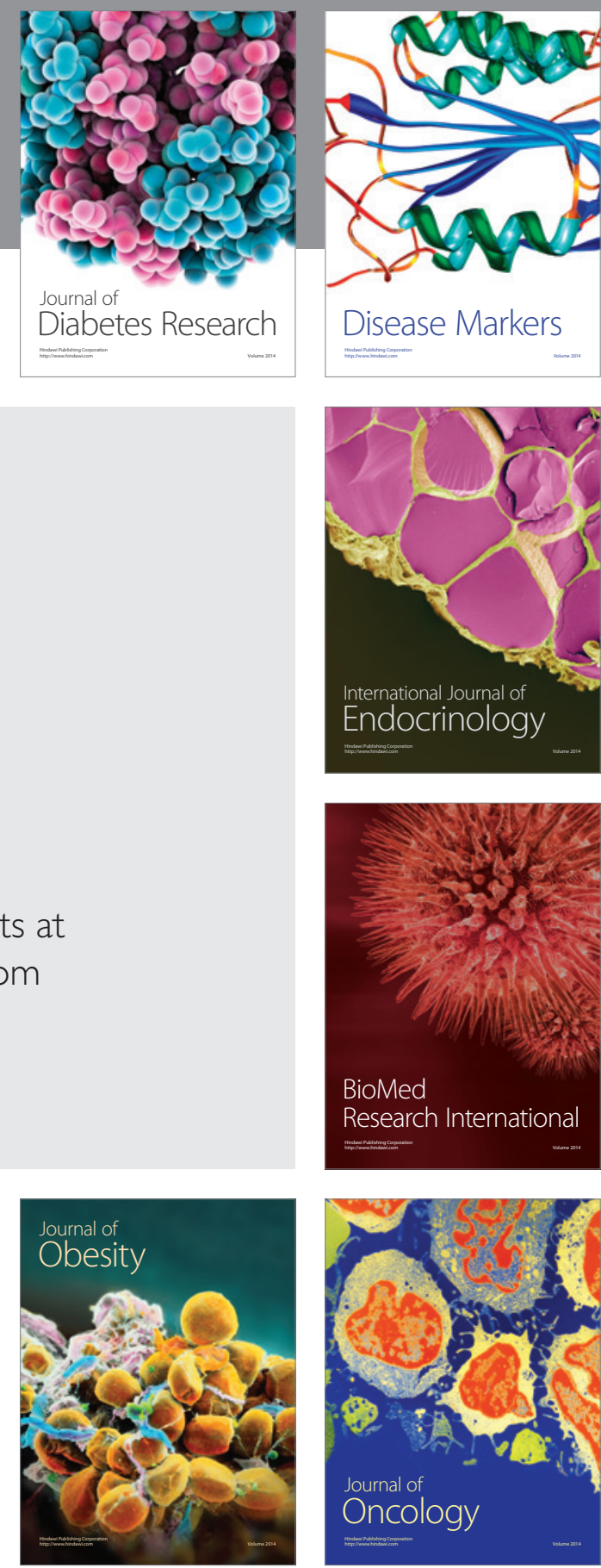

Disease Markers
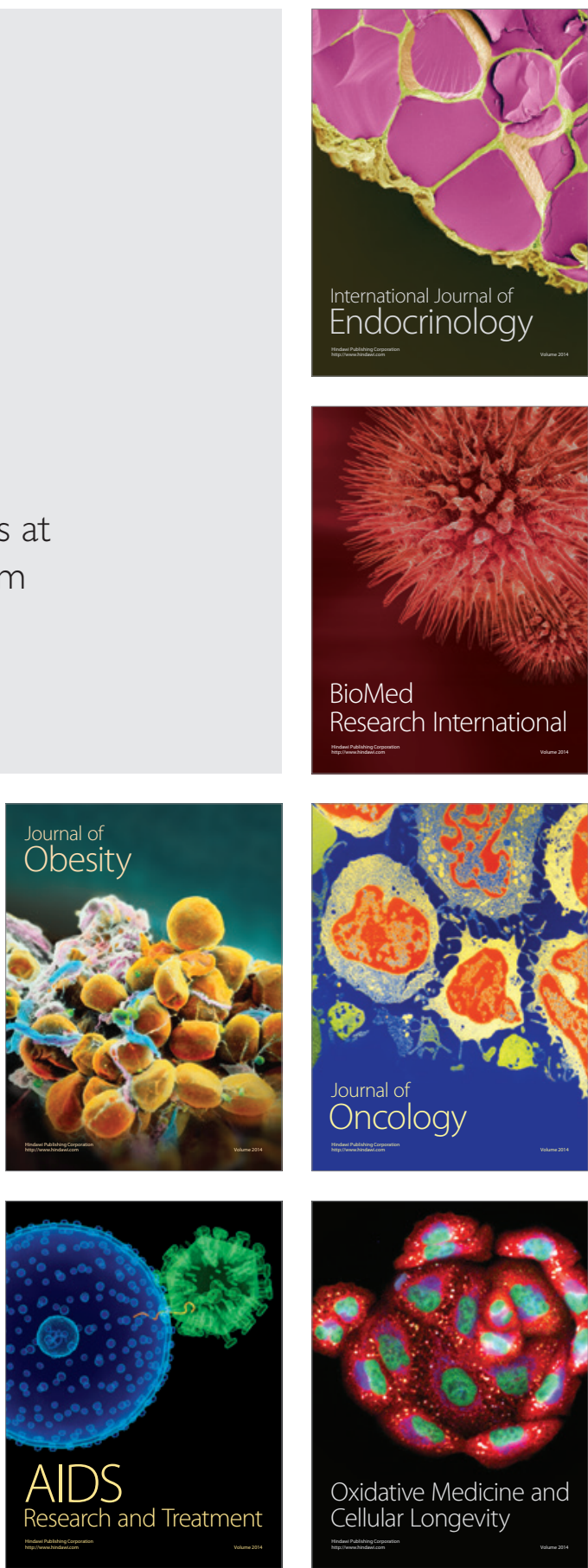